\title{
Three-year experience of prophylactic placement of inferior vena cava filters in women with gynecological cancer
}

This article was published in the following Dove Press journal:

International Journal of General Medicine

12 August 2013

Number of times this article has been viewed

\author{
Suresh B Babu' \\ Asiya Maheen Khan ${ }^{2}$ \\ Philip JB Coates' \\ 'Radiology Department, Derriford \\ Hospital, Plymouth, UK; ${ }^{2}$ Radiology \\ Department, City Hospital, \\ Birmingham, UK
}

Objective: To evaluate the outcomes of prophylactic placement of inferior vena cava (IVC) filters to prevent pulmonary embolism (PE) in women undergoing surgery and chemotherapy for gynecological cancer.

Methods and materials: Thirty-eight IVC filters were placed in 38 women between January 2008 and January 2010; 25 of these were placed in gynecological cancer patients for prevention of PE during surgery and the postoperative period. The patients' electronic medical records, follow-up computed tomography scans, and outpatient follow-up notes were retrospectively reviewed for incidence of PE and adverse events.

Results: After 6 months of follow-up, no PE was observed and there was no mortality. Nine filters were retrieved uneventfully, and there were no clinical complications associated with any indwelling filter.

Conclusion: IVC filters are safe and beneficial towards preventing PE in women undergoing surgery and chemotherapy for gynecological cancer.

Keywords: inferior vena cava filter, gynecological cancer, prophylaxis, pulmonary embolism

\section{Introduction}

It is well recognized that women with gynecologic malignancies are at high risk of developing venous thromboembolism (VTE; ie, deep vein thrombosis [DVT] or pulmonary embolism [PE]). ${ }^{1-4}$ These women are associated with several contributing risk factors: malignancy, major pelvic/abdominal surgery, chemotherapy treatment, and often advanced age and chronic medical comorbidities. In the absence of preventative measures, the prevalence of DVT among women undergoing major gynecologic surgery is estimated to be $15 \%-40 \% .{ }^{5}$ Moreover, postoperative PE is a leading cause of death for this patient population. ${ }^{3}$

The American College of Chest Physicians guidelines (9th edition) recommend routine thromboprophylaxis in all women undergoing surgery for gynecological malignancies, except in very low-risk groups for VTE. Specifically, anticoagulation, intermittent pneumatic compression, and graduated compression stockings, or a combination thereof, are recommended for 28 days in this patient population above low risk for VTE; prophylaxis may be tailored to individual patient needs, based on their individual risk factors for VTE. ${ }^{1,5}$ When used, postoperative pharmacological prophylaxis is associated with a significant reduction in the incidence of postoperative VTE. ${ }^{1,4}$ Moreover, prolonged prophylaxis may be important, as at least one institution has observed that $75 \%$ of VTE events occurred more than 7 days following surgery for gynecological cancer and $36 \%$ occurred 4 weeks after the procedure. ${ }^{6}$ In fact,
Correspondence: Suresh B Babu Interventional Imaging, Radiology Department, Derriford Hospital, Derriford Road, Crownhill, Plymouth, Devon PL6 8DH, UK

Tel +44789485 6490 Email drsureshbabu@hotmail.com 
prolonged anticoagulation was associated with a $78 \%$ reduction in VTE events through 30 days following surgery for gynecologic cancer ${ }^{7}$; others have reported similar findings in patients undergoing major abdominal surgery or surgery for abdominal or pelvic cancer. ${ }^{8,9}$ Even with pharmacological prophylaxis, a 3-month PE rate of $1.1 \%$ was recently reported for gynecological cancer patients; moreover, anticoagulation was associated with undesired hemorrhagic complications in these patients. ${ }^{10}$ Concerns of such complications may in part explain the reported low use of pharmacological prophylaxis. $^{2}$

Therefore, inferior vena cava (IVC) filters may have a role in the treatment of gynecological oncology patients. Filters may be especially useful in women with complications or contraindications to, long-term anticoagulation or women with multiple risk factors for PE. However, there are limited data regarding filter use in this patient population. ${ }^{11,12}$ This retrospective analysis was performed specifically to evaluate the safety and effectiveness of IVC filter placement as prophylaxis against PE in women undergoing surgery for gynecological cancer.

\section{Methods and materials}

This was a single-center, retrospective review of patients receiving IVC filters for PE prophylaxis before undergoing surgery for gynecological malignancies. Patients treated at our hospital between January 2008 and January 2010 were identified from a prospectively maintained hospital database. Data through 6-month postplacement were reviewed, including electronic medical records, follow-up computed tomography (CT) scans (including of the chest), and outpatient follow-up notes for the occurrence of pulmonary embolism, advances in the cancer stage, adverse events, or death. Ethics committee approval was not required because of the retrospective nature of the study.

Patients received filters as day cases prior to the planned surgery for their gynecological malignancy. The initial CT scan of the abdomen and pelvis (obtained primarily to investigate the gynecological malignancy) was evaluated for the presence of any IVC thrombus or iliac venous thrombus, the diameter of the IVC, and any possible anomalies of the IVC (eg, double IVC). Patients had provided written informed consent prior to the procedure.

Filters were placed under local anesthesia, and generally via jugular access. Once access was obtained, a flush venogram was done to evaluate the IVC and the level of the renal veins. A Günther Tulip IVC filter (Cook Medical, Bloomington, IN, USA) was placed in each patient.
The Günther Tulip filter is considered an optional filter, and may be retrieved or left in situ as a permanent filter. The IVC filter was introduced through an $8.5 \mathrm{~F}$ coaxial system. Once the filter was in place, just below the renal veins, confirmation venography was performed. Patients were usually discharged within 4 hours.

\section{Results \\ Patient demographics}

In total, 38 IVC filters were placed in women during the study period. Twenty-five women (mean age $56 \pm 7$ years) received an IVC filter as prophylaxis for PE prior to surgery for a gynecological malignancy (this bias is due to the fact that the hospital is a large tertiary referral center for gynecological malignancies). Most of the women were British in origin (75\%), and all had advanced-stage cancer. Malignancies included ovarian cancer $(n=19)$, uterine sarcomas $(n=4)$, and cervical cancer $(n=2)$. More than half of the women (68\%) had either already received chemotherapy or were receiving chemotherapy at the time of filter placement. Baseline demographics are summarized in Table 1.

\section{Filter placement}

Most patients received filters as permanent devices $(n=14)$. Filters were placed uneventfully, with no access-site problems, bleeding during procedure, or immediate postprocedural complications. Two patients had a filter tilt of more than $15^{\circ}$ on completion. There were no immediate deaths

Table I Baseline patient demographics

\begin{tabular}{ll}
\hline Characteristic & $\mathbf{n}$ \\
\hline Age (years) & $56 \pm 7$ \\
Ethnicity & 18 \\
British & 2 \\
Afro-Caribbean & 2 \\
Asian & 3 \\
Not reported & \\
Type of gynecologic cancer & $76 \%$ \\
Ovarian carcinoma & $16 \%$ \\
Uterine sarcoma & $8 \%$ \\
Cervical cancer & \\
Ovarian cancer stage & 0 \\
I & 0 \\
II & 15 \\
IIlc & 4 \\
IV & 8 \\
Previous DVT & \\
Filter planned as & \\
Permanent & 14 \\
Temporary & 11 \\
\hline
\end{tabular}

Abbreviation: DVT, deep vein thrombosis. 
from IVC filter insertion. Anticoagulation whilst the filter was in situ was variable depending on patient comorbidities. All patients were discharged as day cases.

\section{Filter retrieval}

Eleven patients (44\%) underwent a filter-retrieval attempt once their surgery, hospitalization, and chemotherapy were completed. Nine filters ( $82 \%$ ) were uneventfully retrieved, and two filters could not be removed due to excessive filter tilt and an inability to capture the filter. Other than the failed retrievals, there were no complications associated with filter retrieval.

\section{Complications}

A 6-month follow-up CT was performed for cancer staging postchemotherapy, and 16 patients still had indwelling IVC filters at the time of their imaging. In one patient, the CT revealed two filter legs protruding out of the IVC; the two filter legs extended less than $3 \mathrm{~mm}$ outside the wall of the IVC. The patient was asymptomatic, and no additional events were discerned. No other complications were observed, including IVC occlusions, thrombosis, or filter migration.

\section{Survival}

At 6-month follow-up, all 25 women were alive (ie, the mortality rate was 0 ). There were no PEs or DVTs within the 6 months after filter placement.

\section{Discussion}

IVC filters are used to protect against PE in a variety of patient populations, including those with VTE, cancer, and traumatic injuries, and those undergoing bariatric or orthopedic surgery, among others who have high risks with anticoagulation therapy. Approximately half of all filters are placed to prevent PE in patients without a history of VTE but considered at high risk for developing PE. The effectiveness of IVC filters in preventing PE has been demonstrated previously. Most notably, the randomized PREPIC study, ${ }^{13,14}$ which enrolled patients with existing proximal DVT, demonstrated that patients receiving a filter were associated with a decreased incidence of PE compared to patients that did not receive a filter, and these patients were also given anticoagulation; however, patients with filters were associated with an increased incidence of DVT in longer follow-up (ie, 2 and 8 years). There is also a wide range of reports on IVC filter performance in patients receiving filters for a variety of clinical conditions, including cancer, considered to put them at high risk for PE; in general, the literature is supportive of IVC filter safety and efficacy towards preventing PE. ${ }^{11,12,15-17}$
The potential risks of IVC filter placement are well documented. ${ }^{18,19}$ In general, indwelling IVC filters are associated with low complication rates, although patients with filters in place for prolonged periods may be more likely to experience such complications as DVT, filter fracture, IVC perforation, and others. Therefore, the potential risks of filter placement must be considered along with the potential risk for VTE and the potential clinical benefits of filter placement on a patient-by-patient basis, and similarly for filter retrieval. In cancer patients, this includes considerations for the stage of the disease and the life expectancy of the patient. ${ }^{20}$

Women with gynecologic malignancies have multiple risk factors for DVT and PE, and PE is a major cause of death in these patients. While prophylaxis for PE in these patients has traditionally been provided via anticoagulation, intermittent pneumatic compression, graduated compression stockings, or a combination thereof, these therapies may not be appropriate or sufficient for all patients. Thus, IVC filter placement may be beneficial in this patient population. In fact, two previous studies have reported safe and effective use of IVC filters in women with gynecological malignancies. ${ }^{11,12}$

Results of this single-center, retrospective analysis support the results of previous studies and suggest that IVC filters are safe and beneficial in preventing PE and PE-related deaths in women undergoing surgery for gynecological malignancies. Our patient population included women with ovarian, uterine, and cervical malignancies; the majority of patients were treated for ovarian cancer, a patient group that is associated with a higher incidence of VTE than other gynecologic malignances. ${ }^{21}$ There were no findings of PE or DVT in follow-up, and there were few filter-related complications. Among eleven patients in whom filter retrieval was attempted, nine $(82 \%)$ filters were successfully retrieved. The decision to retrieve a filter was based upon cancer status, chemotherapy status, time since filter implantation, and life expectancy for each patient. For most patients, filters were placed as permanent devices because of ongoing cancer treatment and a limited life expectancy. It is possible that retrievable optional IVC filters may have higher complication rates than permanent filters in the long term.

Limitations of this study include its single-center, retrospective nature. In addition, the study included a small number of patients. Further studies with a larger number of patients or randomized controlled trials would also be useful in confirming our findings. The study included only a limited follow-up period, although this may be appropriate for this patient population, as their life expectancy may be reduced compared to the majority of patients receiving filters. 
Moreover, follow-up imaging was dependent on the patient's follow-up as related to their cancer, and was not specifically focused on the filter. Despite these limitations, the results confirm those of two previous publications and add to the limited available data regarding filter placement in this patient population.

In conclusion, this short-term study demonstrates that optional IVC filters can be safely used to help prevent PE in women undergoing surgery for gynecological malignancies.

\section{Acknowledgments}

The authors thank Jennifer A McCann-Brown, PhD, of the MED Institute (a contract research organization and Cook Group company) for technical assistance with manuscript preparation.

\section{Disclosure}

The authors report no conflicts of interest in this work.

\section{References}

1. Gould MK, Garcia DA, Wren SM, et al. Prevention of VTE in Nonorthopedic Surgical Patients: Antithrombotic Therapy and Prevention of Thrombosis, 9th ed. American College of Chest Physicians evidencebased clinical practice guidelines. Chest. 2012;141:e227S-e277S.

2. Martino MA, Williamson E, Rajaram L, et al. Defining practice patterns in gynecologic oncology to prevent pulmonary embolism and deep vein thrombosis. Gynecol Oncol. 2007;106:439-445.

3. Clarke-Pearson DL, Jelovsek FR, Creasman WT. Thromboembolism complicating surgery for cervical and uterine malignancy: incidence, risk factors, and prophylaxis. Obstet Gynecol. 1983;61:87-94.

4. Einstein MH, Pritts EA, Hartenbach EM. Venous thromboembolism prevention in gynecologic cancer surgery: a systematic review. Gynecol Oncol. 2007;105:813-819.

5. Guyatt GH, Akl EA, Crowther M, et al. Executive Summary: Antithrombotic Therapy and Prevention of Thrombosis, 9th ed. American College of Chest Physicians evidence-based clinical practice guidelines. Chest. 2012;141:7S-47S.

6. Peedicayil A, Weaver A, Li X, Carey E, Cliby W, Mariani A. Incidence and timing of venous thromboembolism after surgery for gynecological cancer. Gynecol Oncol. 2011;121:64-69.

7. Schmeler KM, Langley G, Cain K, et al. Venous thromboembolism (VTE) rates following the implementation of extended duration prophylaxis for patients undergoing surgery for gynecologic malignancies. Gynecol Oncol. 2013;128:204-208.
8. Rasmussen MS, Jorgensen LN, Wille-Jorgensen P, et al. Prolonged prophylaxis with dalteparin to prevent late thromboembolic complications in patients undergoing major abdominal surgery: a multicenter randomized open-label study. J Thromb Haemost. 2006;4:2384-2390.

9. Bergqvist D, Agnelli G, Cohen AT, et al. Duration of prophylaxis against venous thromboembolism with enoxaparin after surgery for cancer. N Engl J Med. 2002;346:975-980.

10. Hitos K, Wain GV, Fletcher JP. Venous thromboembolism following gynecological surgery for suspected or confirmed malignancy. Aust $N$ Z J Obstet Gynaecol. 2012;52:23-27.

11. Dewdney SB, Benn T, Rimel BJ, et al. Inferior vena cava filter placement in the gynecologic oncology patient: a 15-year institutional experience. Gynecol Oncol. 2011;121:344-346.

12. Adib T, Belli A, McCall J, et al. The use of inferior vena caval filters prior to major surgery in women with gynaecological cancer. $B J O G$. 2008;115:902-907.

13. Decousus H, Leizorovicz A, Parent F, et al. A clinical trial of vena caval filters in the prevention of pulmonary embolism in patients with proximal deep-vein thrombosis. N Engl J Med. 1998;338:409-415.

14. PREPIC study group. Eight-year follow-up of patients with permanent vena cava filters in the prevention of pulmonary embolism: the PREPIC (Prévention du Risque d'Embolie Pulmonaire par Interruption Cave) randomized study. Circulation. 2005;112:416-422.

15. Smouse HB, Rosenthal D, Thuong VH, et al. Long-term retrieval success rate profile for the Günther Tulip vena cava filter. J Vasc Interv Radiol. 2009;20:871-877.

16. Given MF, McDonald BC, Brookfield P, et al. Retrievable Gunther Tulip inferior vena cava filter: experience in 317 patients. $J$ Med Imaging Radiat Oncol. 2008;52:452-457.

17. Damascelli B, Ticha V, Patelli G, et al. Use of a retrievable vena cava filter with low-intensity anticoagulation for prevention of pulmonary embolism in patients with cancer: an observational study in 106 cases. J Vasc Interv Radiol. 2011;22:1312-1319.

18. Caplin DM, Nikolic B, Kalva SP, Ganguli S, Saad WE, Zuckerman DA. Quality improvement guidelines for the performance of inferior vena cava filter placement for the prevention of pulmonary embolism. J Vasc Interv Radiol. 2011;22:1499-1506.

19. American College of Radiology. ARC-SIR 2010 practice guideline for the performance of inferior vena cava (IVC) filter placement for the prevention of pulmonary embolism. Revised 2010 (Resolution 46). Available from: http://www.acr.org/ /media/ACR/Documents/PGTS/ guidelines/IVC_Pulmonary.pdf. Accessed May 31, 2013.

20. Abdel-Razek H, Mansour A, Ismael Y, Abdulelah H. Inferior vena cava filters in cancer patients: to filter or not to filter. Ther Clin Risk Manag. 2011;7:99-102.

21. Wang X, Fu S, Freedman RS, Kavanagh JJ. Venous thromboembolism syndrome in gynecological cancer. Int J Gynecol Cancer. 2006;16 Suppl 1: 458-471.
International Journal of General Medicine

\section{Publish your work in this journal}

The International Journal of General Medicine is an international, peer-reviewed open-access journal that focuses on general and internal medicine, pathogenesis, epidemiology, diagnosis, monitoring and treatment protocols. The journal is characterized by the rapid reporting of reviews, original research and clinical studies across all disease areas.

\section{Dovepress}

A key focus is the elucidation of disease processes and management protocols resulting in improved outcomes for the patient.The manuscript management system is completely online and includes a very quick and fair peer-review system. Visit http://www.dovepress.com/ testimonials.php to read real quotes from published authors. 